\title{
Cognitive maps: Analysis of comparative judgments of distance
}

\author{
DAVID R. BAUM \\ Honeywell Systems and Research Center, Minneapolis, Minnesota 55413 \\ and \\ JOHN JONIDES \\ University of Michigan, Ann Arbor, Michigan 48104
}

\begin{abstract}
Subjects were required to judge which of two straight-line distances was shorter in the context of a speeded-response task. In the "memory" condition of the experiment, these distances corresponded to imagined distances between geographic landmarks; in the "perception" condition, the distances were displayed visually for subjects to examine. The data were analyzed by examining patterns of latencies and errors as a function of the similarities between the two distances on each trial. These data suggest that different mechanisms mediate the comparison of distances retrieved from memory as compared to perceived distances.
\end{abstract}

Most people typically behave as if they have quite a bit of knowledge about their spatial environments, unless these environments are particularly unusual in structure (e.g., see Lynch, 1960, for a comparison of representations of various kinds of urban spatial layouts). For example, they can effectively find their way from one place to another that is not in immediate view; they can provide and comprehend directions for traveling from one location to another; and they can make reasonably accurate judgments of distance and bearing between locations. These behaviors and others suggest that humans, and other animals as well (see, e.g., Menzel, 1973; Olton, 1977; Tolman, 1948), possess knowledge structures that preserve information about the layout of environmental space with great fidelity. What kind of information is preserved in such knowledge structures, and how is it accessed?

Casual observation and careful experimentation both indicate that one of the properties of natural spaces to which people are quite sensitive is distance. For example, one is usually able to judge at least roughly how long it would take to travel by foot between, say, two buildings at opposite ends of a familiar university campus. In fact, various experiments have demonstrated that subjects are remarkably consistent in judging distances, in the sense that judgments of distance result

This research was supported in part by a Rackham Faculty Research Grant to John Jonides. The research was conducted while the first author was a predoctoral trainee supported by NIH Grant 5 T32 MH14254-02. We wish to thank James Egan and Peter Ward for invaluable assistance in data analysis and Barbara Zeeff for help in running subjects. Finally, we gratefully thank David Krantz for some insightful discussions and suggestions about the interpretation of the data in this experiment. in a strikingly linear relationship between estimated and actual distance (e.g., Cadwallader, 1976; Canter \& Tagg, 1975; Golledge \& Zannaras, 1973).

One example of the fidelity of distance estimates comes from a study in our laboratory (Jonides \& Baum, Note 1). We demonstrated that even under speeded response conditions, estimates of distance between landmarks on the University of Michigan campus correlated .95 with the actual distances. Furthermore, multidimensional scaling revealed a remarkably good correspondence between the maps recovered by scaling solutions of the distance estimates and an actual cartographic map of the same landmarks. These results show clearly that distance information is well preserved in memory, at least for such environments.

Given this, one might question how distance information is represented and accessed in the course of performing such tasks as distance estimation. Our earlier study suggests one possible hypothesis (Jonides \& Baum, Note 1). We found a substantial linear relationship between distance estimates and the amount of time subjects took to arrive at these estimates. This raises the hypothesis that subjects arrive at their estimates by some sort of analog process that makes use of a mental representation that preserves metric distance data. One might imagine such a mental representation to be something like a "mental map." The estimation process, then, might involve mentally traversing from one location to another, or, perhaps, iteratively laying out a "mental ruler" between landmarks.

These potential strategies (as well as others that are consistent with the latency data) share a strong "perceptual" flavor. That is, they draw heavily on an analogy to processes that could be involved in estimating distances on an actual cartographic map. In fact, Hartley 
(1977) has proposed a similar class of mechanisms to account for a linear relationship between estimation time and distance that he found when subjects judged perceived line lengths. This, of course, raises the intriguing possibility that processing information from maps stored in memory is quite similar to processing information from perceived maps.

How could one further test this hypothesis? The most direct test would involve an explicit comparison of processing characteristics between conditions in which the map information is either stored in memory or directly presented visually. A comparison of our earlier work (Jonides \& Baum, Note 1) with that of Hartley (1977) suggests that the analogy between perceptual and memorial conditions is a potentially productive one. In the present experiment, we sought to extend this analogy to a task that required the use of distance information, but not in the context of estimation. Subjects in this experiment were presented a pair of landmarks on each trial and asked to judge which member of the pair was closer to a reference landmark. In a "perception" condition, the landmark locations were visually presented at the time of judgment; in a "memory" condition, subjects were required to recall the landmark locations from memory on the basis of their knowledge of a highly familiar space.

What would one expect about performance in the memory condition if subjects operated on a representation that shared important similarities with the representation available in the perception condition? The obvious prediction is that performance in the memory condition would be similar to that in the perception condition. But in what ways? Surely one would not be surprised to find sizable differences in overall latency and accuracy between the two conditions (in some obvious sense, having the information actually present should make the perceptual task easier). The analysis of interest goes further than this by comparing patterns of latencies and errors between the two conditions. In order to create such patterns, we varied the difficulty of the task within each condition by manipulating the relative proximity of each test landmark to the reference landmark in each pair. In this way we planned to generate functions of latency and accuracy vs. relative proximity for each condition; these functions could then be compared between conditions to assess similarity of processing characteristics.

\section{METHOD}

\section{General Design}

There were two conditions in the experiment. On each trial in the "memory" condition, subjects were required to evaluate from memory which of two landmarks on the University of Michigan campus was closer to a reference landmark. In the perception condition, subjects performed the analogous task on distances proportional to those used in the memory condition. The difference was that, in the perception condition, the landmarks, represented by the locations of single letters, were actually present at the time of judgment. The distances between these locations were scaled to be proportional to the distances in the memory condition.

\section{Subjects}

The subjects were University of Michigan undergraduates of sophomore, junior, or senior standing. They were paid for participation in two sessions (one practice and one test) on separate days. Eight females and five males served in the memory condition; five females and five males served in the perception condition. All subjects were screened for familiarity with the locations of the 10 landmarks that were used as stimuli in the memory condition.

\section{Apparatus and Stimuli}

An IBM 1800 computer was used to control stimulus presentation and to collect data on both the identity and the latency of each response. Subjects were seated so that their eyes were approximately $50 \mathrm{~cm}$ from the CRT screen on which the stimuli were displayed.

The stimuli in the memory condition were the names of 10 campus landmarks. These particular landmarks were chosen because they were highly familiar and because they adequately sampled a space in which the longest distance was approximately $1,900 \mathrm{ft}$, and the shortest, approximately $100 \mathrm{ft}$. The stimuli for the perception condition were 10 locations on the CRT screen; they were chosen such that if a campus map drawn to the scale of the CRT screen were superimposed over the screen, the centers of the 10 landmarks of the memory condition would overlay the 10 screen locations of the perception condition. All 10 locations in the perception condition are presented in Figure 1, which is drawn to scale. The corresponding landmark names of the memory condition are also indicated in the figure (although the landmark names did not actually appear in the perception condition). The minimum interpoint distance between locations in the perception condition was $26 \mathrm{~mm}$ and the maximum was $167 \mathrm{~mm}$ (18.5 deg visual angle).

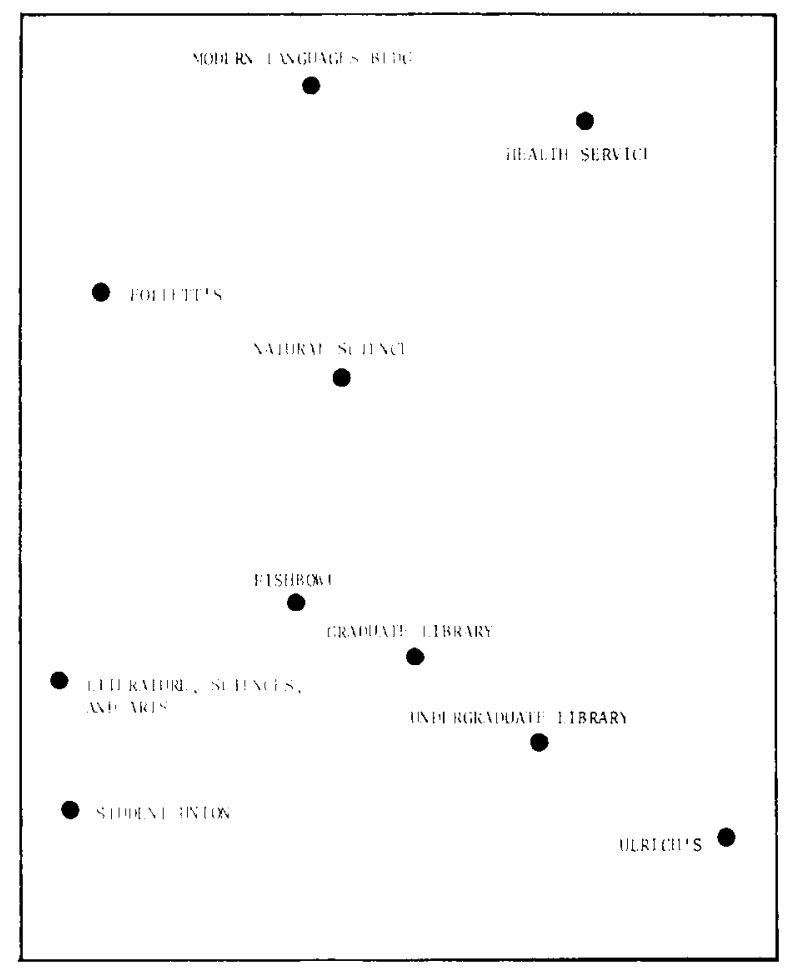

Figure 1. Replica of the relative visual display locations used in the perception condition. Locations are indicated by filled circles. Building names are those used in the memory conditions; however, the names were displayed one below the other in the center of the screen. Only three names or locations were displayed at any one time. 


\section{Procedure}

Memory condition. The sequence of events on each trial was as follows: The word "ready" was first displayed in the center of the screen for $1.0 \mathrm{sec}$. Then a "plus" sign appeared in the center of the screen, followed by 1 of the 10 landmark names (this was the reference point). The reference landmark alone remained in view until the subject pressed one of two response buttons. Following a delay of $1.0 \mathrm{sec}$, two other landmark names (the anchor landmarks) appeared immediately below the reference landmark. If the subject judged the top anchor landmark to be closer to the reference landmark (that is, actually closer to it on campus, not on the screen), he was to depress the left response button; if he judged the bottom anchor landmark to be closer, he was to press the right button. A $3.0-\mathrm{sec}$ intertrial interval elapsed between each response and the next "ready" signal. The word "error" was displayed during the first $1.0 \mathrm{sec}$ of this interval if the subject had judged the wrong anchor to be closer to the reference point; otherwise, the CRT screen was blank during the intertrial interval. The anchors were arranged so that $50 \%$ of the time the top one was correct, and $50 \%$ of the time the bottom one was correct.

Perception condition. The events for this condition were identical to those of the memory condition except for the following: When the "plus" sign appeared after the word "ready," it was positioned at the location of the impending reference landmark. This reference landmark then appeared as the letter "O," replacing the "plus" sign. When the subject initiated the presentation of the anchors, the anchors appeared in their appropriate locations on the screen. One anchor (randomly selected) was represented by the letter "L," the other by the letter "R." Subjects were then required to depress the left or the right button if they judged the $L$ or the $R$, respectively, to be closer to the $O$. Subjects in this condition were told that the locations at which the letters appeared in fact represented campus landmarks, although they would not actually need this information to perform the task accurately.

For each condition, there were 120 practice trials run in a 30 min session and 360 test trials run in another session of $1 \mathrm{~h} 30 \mathrm{~min}$. For the 360 test trials, each landmark served as the reference 36 times. The two anchors that appeared with each reference landmark were chosen from the nine remaining landmarks using all possible combinations (with one instance only of each anchor pair). These 360 trials were randomized and divided into three blocks of 120 trials each. The blocks were run with 5 -min rest intervals intervening.

The instructions given before the practice and test trials emphasized the following points: All of the comparative judgments were to be based on the straight-line distances between the closest points of each anchor and the reference landmark. All of the judgments were to be made as rapidly but as accurately as possible. In order to promote optimal responding, subjects were instructed not to initiate presentation of the anchors on each trial until they were prepared for the comparative judgment task.

\section{RESULTS}

\section{Overall Performance}

As expected, subjects in the perception condition responded faster and with fewer errors than did subjects in the memory conditions ( 951 vs. $2,822 \mathrm{msec}$ and $5.1 \%$ vs. $16.3 \%$ errors, respectively). These differences between conditions in latency and accuracy are highly reliable $[\mathrm{t}(21)=8.19, \mathrm{p}<.001 ; \mathrm{t}(21)=6.63, \mathrm{p}<.001$, respectively]. Of course, as discussed above, it is not particularly surprising to find such differences in overall performance. In the perception condition, all the information is present on the screen at the time of judgment, whereas in the memory condition, the landmark locations must be retrieved from memory before a judgment can be made. It seems reasonable to suppose that perceptual encoding is both a faster and a more accurate process than memory retrieval.

\section{Patterns of Performance}

The more interesting data from the experiment concern the relative patterns of latencies and errors vs. judgment difficulty that were obtained in each condition. In order to evaluate these patterns, we calculated various linear and quadratic regressions of average latencies and error rates for both conditions as a function of various measures of the physical space represented by the stimuli. ${ }^{1}$ Table 1 lists the percentages of variance accounted for in each of these regressions for both conditions and both dependent variables. The variables used as predictors in these regressions were: (1) the shorter of the two reference-point-to-anchor distances, $S ;(2)$ the longer of the two reference-point-to-anchor distances, $\mathrm{L} ;(3)$ the sum of these two distances, $\mathrm{S}+\mathrm{L}$; (4) the difference between the two distances, $\mathrm{L}-\mathrm{S} ;(5)$ the ratio of the shorter to the longer distance, $\mathrm{S} / \mathrm{L}$; and (6) the angle, $\mathrm{A}$, formed by connecting one anchor to the reference point to the other anchor. We turn now to an examination of the patterns of performance in both conditions.

Memory condition. On the basis of the analyses indicated in Table 1, there appear to be two variables that account for a substantial portion of the variance in both latency and errors: $L-S$ and $S / L$. Two other variables, $S$ and $S+L$, account for a large proportion of

Table 1

Percentages of Variance Accounted for as a Result of Linear (L) and Quadratic (Q) Regressions of Latency and Errors in Both Experimental Conditions

\begin{tabular}{|c|c|c|c|c|c|c|c|c|}
\hline \multirow[b]{3}{*}{ Predictor Variable } & \multicolumn{4}{|c|}{ Memory Condition } & \multicolumn{4}{|c|}{ Perception Condition } \\
\hline & \multicolumn{2}{|c|}{ Latency } & \multicolumn{2}{|c|}{ Errors } & \multicolumn{2}{|c|}{ Latency } & \multicolumn{2}{|c|}{ Errors } \\
\hline & $\mathbf{L}$ & $\mathbf{Q}$ & $\mathbf{L}$ & Q & $\mathbf{L}$ & Q & $\mathbf{L}$ & $\mathbf{Q}$ \\
\hline Shorter distance (S) & $37.9 *$ & $40.2 \dagger$ & $7.8^{*}$ & 7.8 & $19.8^{*}$ & 20.0 & $3.8^{*}$ & 3.8 \\
\hline Longer distance (L) & .8 & 1.2 & $8.2^{*}$ & 8.9 & 1.0 & 1.2 & $2.5^{*}$ & $3.3+$ \\
\hline Shorter distance + longer distance $(S+L)$ & $14.8 *$ & 14.9 & .0 & 1.7 & $3.2^{*}$ & $5.2 \dagger$ & .0 & 1.1 \\
\hline Longer distance - shorter distance $(L-S)$ & $31.8^{*}$ & 32.6 & $40.1^{*}$ & $55.6 \dagger$ & $34.3^{*}$ & $48.4 \dagger$ & $15.0^{*}$ & $25.5 \dagger$ \\
\hline Shorter distance/longer distance (S/L) & $51.7 *$ & 52.0 & $39.5^{*}$ & $49.2 \div$ & $45.4^{*}$ & $58.3 \div$ & $18.7^{*}$ & $29.4 \div$ \\
\hline Angle of one anchor to reference point to other anchor (A) & $3.5^{*}$ & 3.6 & .9 & .9 & .4 & $4.1 \dagger$ & 1.6 & 3.4 \\
\hline
\end{tabular}

${ }^{*} p<.01$ (all other entries are nonsignificant; i.e., $p>.01$ ).

†Significant amount of additional variance accounted for by quadratic regression $(p<.01)$. 
variance in latency, but much less so for errors. Finally, A accounts for a statistically significant amount of variance in latency only, and $L$ is a reliable predictor of errors only. However, the magnitudes of the latter two effects are relatively small, so we shall disregard them. ${ }^{2}$

On the face of it, we seem to have obtained a fairly complex result, since there are so many predictors that appear to account for latency and error variation. However, careful analysis of the data reveals that the situation is actually quite simple. Due to a confounding among variables for the particular landmarks used in the experiment, there is a substantial correlation between the $\mathrm{S} / \mathrm{L}$ variable and each of the others $(.67$ with $\mathrm{S}, .41$ with $S+L$, and .82 with $L-S$ ). In fact, with $S / L$ used as a predictor variable for latency in a linear regression, there is no substantial additional prediction of the residual variance with any of the remaining variables (1.2\% for $\mathrm{S}, 1.8 \%$ for $\mathrm{S}+\mathrm{L}$, and $.1 \%$ for $\mathrm{L}-\mathrm{S})$. In contrast, predicting the residual variance from the linear regressions of the other variables on latency using S/L yields sizable effects $(10.6 \%$ for prediction of the residual variance of $\mathrm{S}, 37.2 \%$ for prediction of the residual variance of $\mathrm{S}+\mathrm{L}$, and $9.8 \%$ for prediction of the residual variance of $L-S$ ). A similar pattern of effects is obtained for the error data with respect to the $\mathrm{S}+\mathrm{L}$ and $S$ variables $(S / L$ accounts for $40.2 \%$ of the remaining variance after $S+L$ is used as a predictor, whereas $S+L$ accounts for only $2.3 \%$ of the residual variance after $S / L$ is used as a predictor; comparable values for the relationship of $S$ and $S / L$ are $19.0 \%$ and $6.1 \%$, respectively). In the case of the difference variable, $L-S$, there was a virtual tie in the comparable scores $(2.3 \%$ vs. $2.0 \%)$. In general, then, it appears that the single variable accounting for most of the variance in latency and in errors is the ratio of the shorter to the longer distance, S/L. Figure 2 presents a scatterplot with a best linear fit for latency as a function of $\mathrm{S} / \mathrm{L}{ }^{3}{ }^{3}$ Figure 3 presents comparable data for errors.

Perception condition. Table 1 reveals that the pattern of results for the regression analyses of latency and error data for the perception condition is quite similar to that for the memory condition. Again, there is a small but reliable effect (for the quadratic fit) of A on latency, of $S+L$ on latency, and of $L$ and $S$ on errors. But the sizable effects are those for the $L-S$ and $S / L$ variables on latencies and errors and for $S$ on latencies. The same confounding among $S, L-S$, and $S / L$ is present in this condition as well as in the memory condition, since the two conditions use the same stimulus space, merely in different scales. Once again, analysis of residual variances reveals that the ratio variable is the basic predictor for all of these effects, as it was in the memory condition. This is supported by the fact that, when both of the variables $S$ and $L-S$ are used as predictors of latency and of errors in linear regression, a reliable and frequently sizable amount of the residual variance is accounted for by $\mathrm{S} / \mathrm{L}$. When $\mathrm{S} / \mathrm{L}$ is used as the original predictor, on the other hand, only a small proportion of the remaining

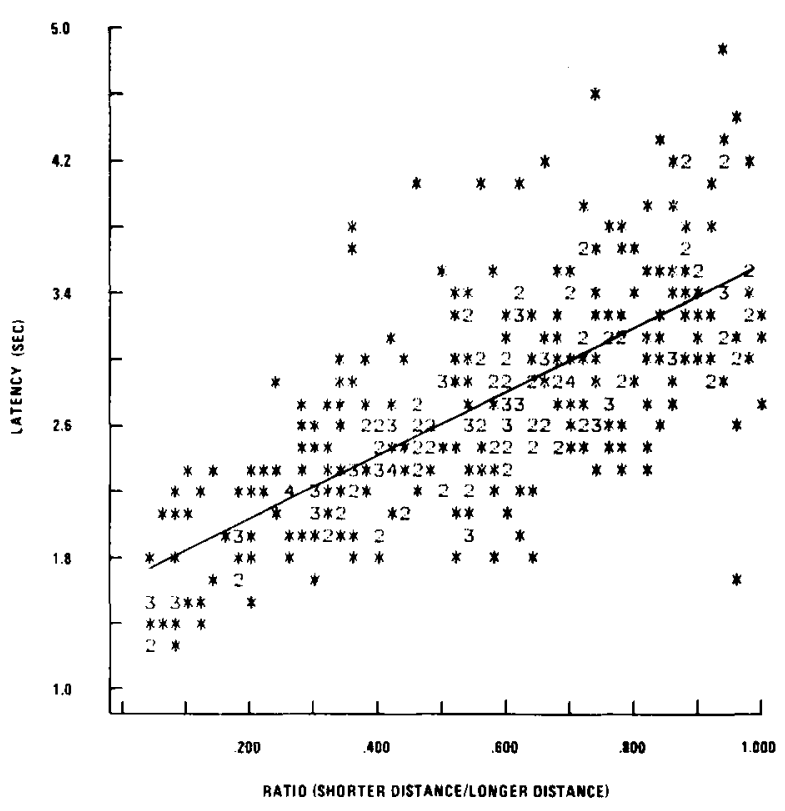

Figure 2. Memory condition: Scatterplot of the relation between distance ratio and mean (correct) latency. Best-fit linear relation is indicated.

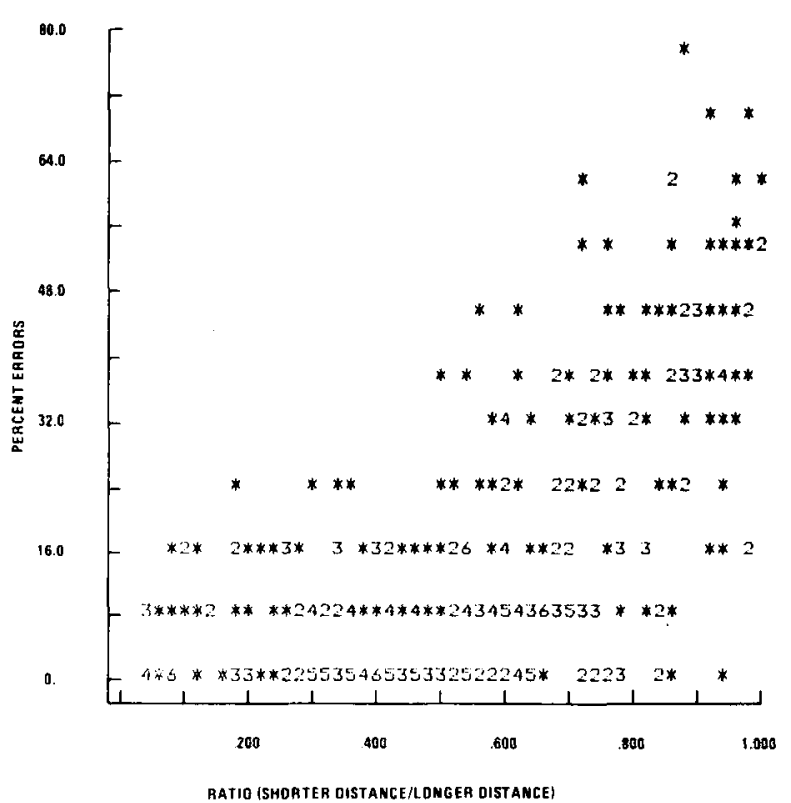

Figure 3. Memory condition: Scatterplot of the relation between distance ratio and percent errors.

variance is accounted for by $\mathrm{S}$ or $\mathrm{L}-\mathrm{S}$. The relevant percentages of variance resulting from this analysis are $21.3 \%$ vs. $2.3 \%$ for $S$ and $15.8 \%$ vs. $7.5 \%$ for $L-S$. Thus, the ratio of the shorter to the longer of the two distances appears to account for more variance in latency and errors than does either of the variables $S$ or $\mathbf{L}-\mathrm{S}$.

Conditions compared. The above account is incomplete, however, as careful examination of Table 1 reveals. In the perception condition, prediction of both latencies and errors by $\mathrm{S} / \mathrm{L}$ is substantially improved by a quadratic. as compared with a linear, function. This is 
not the case in the memory condition: The increase in predictiveness of latency with a quadratic function is not statistically reliable in this condition. The difference between the data of the two conditions is well illustrated by comparing Figures 4 and 5 , which are scatterplots of latency and errors, respectively, vs. S/L for the perception condition, to Figures 2 and 3, the analogous scatterplots for the memory condition. ${ }^{4}$ It is clear from the comparison that, although using $\mathrm{S} / \mathrm{L}$ as a predictor accounts for substantial variance in the perception condition data, the relationship of either latencies or errors to the ratio is quite different in this condition from that in the memory condition. In fact, the latencies of the perception condition are actually much better fit by a power function with an exponent greater than 1 . One such function that describes the latency data quite well is $\ln ($ latency $)=.29[(\mathrm{~L}+\mathrm{S}) /(\mathrm{L}-\mathrm{S})]-.29 .^{5}$ (We did not find a comparable function for the error data, since it is clear from Figure 5 that most of the stimuli yielded error rates of 0 . Nevertheless, casual inspection of the figure suggests the same sort of function as that fit to the data of Figure 4.) This function accounts for $65.9 \%$ of the variance in latency. Comparison of this percentage of variance with that in Table 1 for latency vs. $S / L$ in the perception condition shows a clear improvement in prediction. Moreover, the residual variance remaining after regression with the power function is unsystematic: It does not correlate reliably with the shorter distance, the longer distance, the sum, the difference, the ratio, or the anchor-to-referencepoint-to-anchor angle. Thus, a positively accelerated function seems to yield the most economical description of the perception condition data.

In summary, at a general level, the two conditions yield similar results in that strong distance effects are present in the data: The same measures of distance

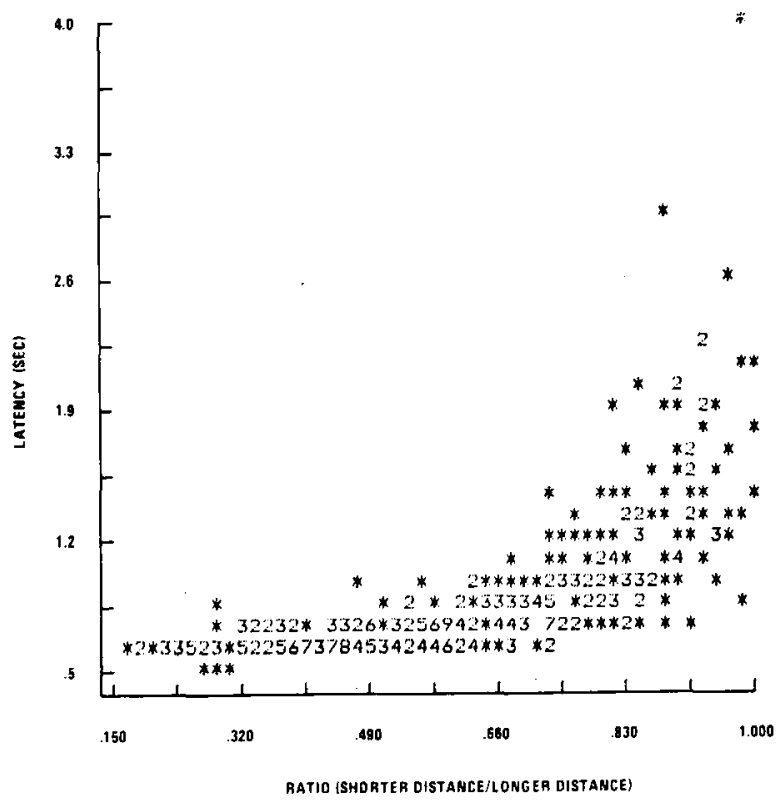

Figure 4. Perception condition: Scatterplot of the relation between distance ratio and mean (correct) latency.

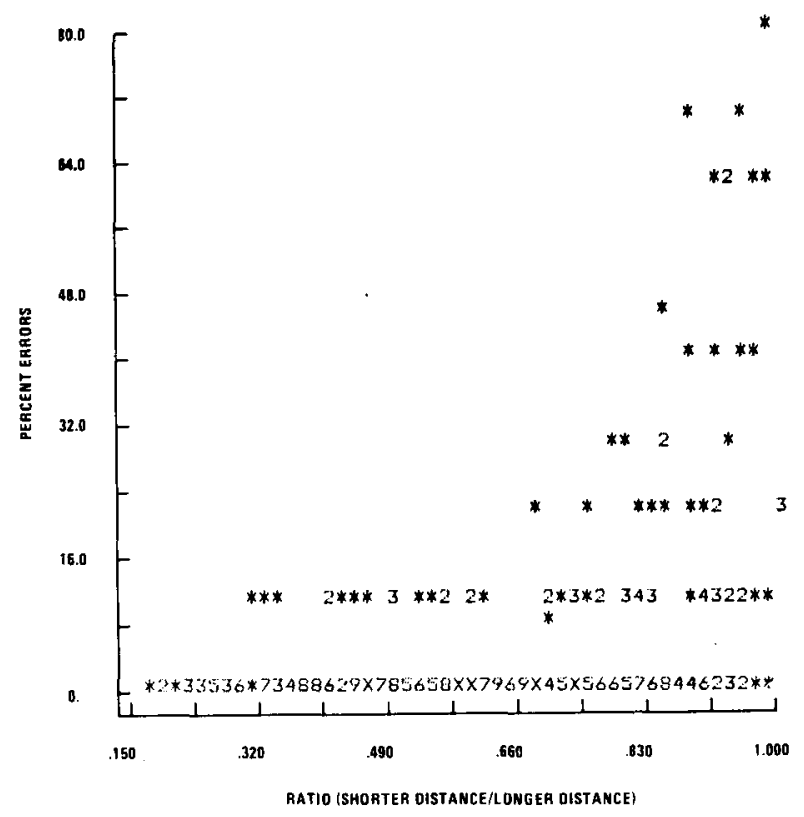

Figure 5. Perception condition: Scatterplot of the relation between distance ratio and percent errors.

account for large percentages of variance in both cases. However, while the results are similar, a detailed comparison of the pattern of predictive relationships reveals a noteworthy distinction: The functions that give the best account of performance are different for the two conditions.

\section{DISCUSSION}

The reported experiment required subjects to compare distance magnitudes drawn either from their memory of the distances involved or from displays in which the distances were immediately available to perception. Comparison of overall performance between the conditions demonstrated clear differences in both latency and error rates. More importantly, however, a comparison of the patterns of latencies and errors vs. judgment difficulty showed that the best predictor of performance was not the same for the two conditions: In the memory condition, performance was most directly related to the ratio of the shorter to the longer distance, while in the perception condition, performance was not a linear function of this ratio.

Before concluding that performance in the two conditions is mediated by different basic comparison processes, we must consider the possibility that some superficial artifact of the experimental procedure is actually the cause of the differences in the performance functions. Two possibilities suggest themselves: First, consider the fact that latencies and errors are much lower overall in the perception than in the memory condition. Performance may have been so good in the perception condition that subjects were limited by a performance ceiling. Such a ceiling effect could have produced the overall shape of the perception condition performance functions. In order to test for this possi- 
bility, we subdivided the data points in Figures 4 and 5 into six equally spaced categories of ratios, and we examined the skewness of the distributions of points within each category for each subject. If the data from the lowest ratios were influenced by a ceiling effect, these distributions should have been more skewed than those for categories of ratios nearer to a value of 1 . In fact, this was not the case: Skewness was unsystematically related to the ratio. Therefore, we must reject the possibility that a ceiling effect contaminated the perception condition data.

Another fairly superficial difference between the perception and memory conditions is that, in the perception condition, eye movements were surely required on at least some trials to encode the two critical lengths about which a decision was required. How would such eye movements influence our performance measures? The most straightforward prediction is that, as the two lengths became more physically separate or as the distance between the anchor landmarks became larger, performance should have deteriorated (at least as measured by latency) because the probability of occurrence of one or more eye movements would increase. These increases in encoding difficulty should be related to two of the variables for which we reported linear and quadratic regressions, $A$ and $S+L$. Examination of Table 1 shows, however, that neither of these variables is a particularly potent predictor of performance. Hence, although eye movements may have occurred during the perception condition, they did not affect judgment latency or accuracy very much.

Having disconfirmed predictions drawn from these more superficial differences, then, we are led to conclude that the performance differences between the experimental conditions reflect basic differences in underlying processes. Our previous experiment involving distance estimation, however, led us to hypothesize that performance might be similar in the perception and memory conditions if subjects were using a similar kind of analog representation and a counting process, albeit implicit, to arrive at their judgments. Has the present experiment disconfirmed our hypothesis about the nature of the representation and processing of distance information?

Clearly, the strongest version of the hypothesis has been falsified (at least insofar as it extends to comparisons of distance in addition to estimates of distance). Comparing distances from memory is not accomplished by the same process as comparing distances that are visually present. ${ }^{6}$ This does not imply that one must abandon the general notion that the memorial representation involved in the magnitude comparison task has an analog quality, nor for that matter that it is accessed by a counting process. In fact, it must be noted that the distance ratio, $\mathrm{S} / \mathrm{L}$, is, in the context of a linear function accounting for latency $\left[a+b\left(S_{S} / S_{L}\right)\right]$, simply a convenient shorthand for writing the Weber ratio, $S /(S+\Delta S)$. Therefore, cognitive comparisons of distance, as measured by judgment latency, do follow a function characteristic of psychophysical judgments. However, the internal representation and comparison process of the memory condition are not identical to those used when the information is available to perception.

Kerst and Howard (1978) have proposed a "reperceptual" model of memory access during distance estimation that may seem, on the face of it, to be consistent with this view. They characterize distance estimates made from memory according to two transformations, one that creates a representation stored in memory at the time that a particular distance is first perceived, and another that occurs when this memory representation is retrieved. These two transformations are assumed to be identical, however, and therein lies the problem in applying this model to the present data. Although the re-perceptual model does claim that representations used for judgments under perceptual and memorial conditions are different (by virtue of having been transformed either once or twice), it fails to explain the qualitative difference in performance that we have obtained in these conditions, given that the two transformations involved are assumed to be identical. So, although this model may be consistent with estimation judgments, it is not a viable explanation of our comparison judgments.

What processes, then, are responsible for comparison judgments in the two experimental conditions? The present experiment offers no unequivocal answers to this question, and, in fact, it suggests no obvious hypothesis for the perception condition. However, the linearity of the memory condition data with ratio invites some speculation about the processes underlying performance in this condition. Consider the possibility that subjects in this condition make use of a mental map similar to the one that we have discussed above. Suppose that, in a first stage of processing, subjects create as clear an image as possible of the campus space containing the three landmarks of a particular trial. Kosslyn (1978) has suggested that this kind of clear image has the property that it is of roughly a constant size. Consistent with this suggestion, we can assume that the images of the landmarks in a triplet are scaled in working memory so that the longer of the two distances is roughly constant from trial to trial. According to this suggestion, the subject does not actively try to equalize the image size on each trial; rather, he tries to create as sharp and as large an image as possible. According to Kosslyn (1978), this has the effect of producing a roughly constant image size (in our experiment, this translates into a roughly constant longer distance size).

After the subject has created an image of the three landmarks, by hypothesis, he applies a counting process to the representation during a second stage of processing to determine the shorter distance. This is accomplished by starting at the imaged reference landmark and simultaneously traversing both paths to each anchor landmark at a constant and equal rate for both traverses. When the first landmark is reached, the subject can terminate 
his counting and make a response appropriate to the landmark that he has reached. Because of the scaling accomplished in Stage 1, the Stage 2 process results in a linear relationship between time and ratio.

Of course, this ad hoc model is largely speculative and incomplete. For example, it fails to provide a ready account of the relationship between error rates and the variable $\mathrm{S} / \mathrm{L}$ (although this result is certainly not inconsistent with the basic proposal), and it is not readily extended to the results of the perception condition. The point is that it is an example of a processing model similar to that hypothesized for judgments of distance magnitude, yet not dependent on the strict assumption that perceptual and memorial representations are identical.

Of course, in a sense, the assumption of a strict isomorphism between perceptual and memorial representations is quite farfetched. As Shepard (1975) and Shepard and Chipman (1970) have effectively argued, it is not quite clear what is implied by such a "firstorder" isomorphism. The research strategy that we have adopted here to extend our earlier work (Jonides \& Baum, Note 1) draws on the concept of a "secondorder" isomorphism. We have attempted to find similarities between the relationships in memorial and perceptual representations (see also Podgorny \& Shepard, 1978, for the report of a similar strategy). In the present case, we found that these functional relation ships are different from each other. In doing so, we have come somewhat closer to understanding the characteristics of cognitive maps.

\section{REFERENCE NOTES}

1. Jonides, J., \& Baum, D. R. Cognitive maps: Analysis of distance estimates. Paper presented at the meeting of the Midwestern Psychological Association, May 1978.

2. Knight, J. L., Howells, R. A., \& Cotter, K. D. Decision latencies in cognitive map utilization. Paper presented at the meeting of the Midwestern Psychological Association, Chicago, May 1978.

\section{REFERENCES}

Cadwallader, M. T. Cognitive distance in intraurban space. In G. T. Moore \& R. G. Golledge (Eds.), Environmental knowing. Stroudsburg, Pa: Dowden, Hutchinson \& Ross, 1976.

CANTER, D., \& TAGG, S. Distance estimation in cities. Environment and Behavior, 1975, 7, 59-80.

Golledge, R. G., \& Zannaras, G. Cognitive approaches to the analysis of human spatial behavior. In W. H. Ittelson (Ed.), Environmental cognition. New York: Seminar Press, 1973.

HaktLey, A. A. Mental measurement in the magnitude estimation of length. Journal of Experimental Psychology: Human Perception and Performance, 1977, 3, 622-628.

Kerst, S. M., \& Howard, J. H., JR. Memory psychophysics for visual area and length. Memory \& Cognition, 1978, 6, 327-335.

Kosslyn, S. M. Measuring the visual angle of the mind's eye. Cognitive Psychology, 1978, 10, 356-389.
Lynch, K. The image of the city. Cambridge, Mass: M.I.T. Press, 1960.

Menzel, E. W. Chimpanzee spatial memory organization. Science, 1973, 182, 943-945.

Outon, D. S. Spatial memory. Scientific American, 1977, 236, 82-98.

Podgorny, P., \& Shepard, R. N. Functional representations common to visual perception and imagination. Journal of Experimental Psychology: Human Perception and Performance, $1978,4,21-35$.

SHEPARD, R. N. Form, formation, and transformation of internal representation. In R. L. Solso (Ed.), Information processing and cognition: The Loyola symposium. Hillsdale, N.J: Erlbaum, 1975.

Shepard, R. N., \& ChIPMAN, S. Second-order isomorphism of internal representations: Shapes of states. Cognitive Psychology, 1970, 1, 1-17.

Tolman, E. C. Cognitive maps in rats and men. Psychology Review, 1948, 55, 189-208.

\section{NOTES}

1. In all of these calculations, we used data averaged over subjects in order to reduce the variability present in each subject's data. We believe that, as in Jonides and Baum (Note 1), a good deal of this variability is due to order effects on sequential trials. Although the present experiment was not designed to reveal such effects systematically, it was designed to render them unsystematic across subjects by providing different random trial orders to different subjects.

2. It should be noted that Knight, Howells, and Cotter (Note 2) have recently reported finding a reliable effect of angle in an experiment similar to ours. They do not report the magnitude of this effect, though, so it is difficult to assess its relative importance at this time. Further investigation of this variable seems to be suggested, however.

3. Although it appears that there are data points at a value of $S / L=1.0$ in Figures $2-5$, this is not so. The largest value of S/L was .99 in both conditions.

4. A comparison between the figures associated with the two conditions reveals small differences in the range of ratios shown. This results from the fact that building extent is taken into account in the memory condition but not in the perception condition. The use of closest-point distances between landmarks for calculations reduces the shortest distances significantly. Thus, in the memory condition, a number of small ratios (below .15) were produced. In the perception condition, point-to-point distances were used for computational purposes.

5. An examination of Figure 4 suggests some heteroscedasticity in the data. In order to check whether the reliable accounts of variance that we reported might be due to this feature, we regressed $\ln ($ latency) on $S / L$. The results of this regression also show a highly reliable $(p<.001)$ linear trend $\left(\mathrm{r}^{2}=.60\right)$ and a highly reliable $(\mathrm{p}<.001)$ quadratic fit $\left(\mathrm{r}^{2}=69\right)$. Thus the analysis is essentially unchanged by logarithmically transforming latency.

6. Of course, a comparison of our earlier experiment with that of Hartley (1977) for perceptual length estimates shows that the latency functions are very similar. This suggests that the estimation process and the representations on which those estimates are made may indeed be quite similar. The present experiment demonstrates a boundary condition on this similarity, however. It does not extend to comparisons of distance.

(Received for publication April 2, 1979; revision accepted August 16, 1979.) 